Homology, Homotopy and Applications, vol.10(1), 2008, pp.237-257

\title{
GROUP HOMOLOGY AND EXTENSIONS OF GROUPS
}

\author{
IOANNIS EMMANOUIL AND INDER BIR S. PASSI
}

(communicated by Jean-Louis Loday)

\begin{abstract}
Using Quillen's description of cyclic cohomology in terms of traces on algebra extensions and Burghelea's computation of the cyclic homology of the group algebra of a group $\Pi$, we derive a description of the homology of $\Pi$ with coefficients in a field of characteristic 0 as the inverse limit of a functor on the category of extensions of $\Pi$.
\end{abstract}

\section{Introduction}

It is well-known that the study of the cyclic cohomology groups $H C^{*}(A)$ of an algebra $A$ over a field $k$ of characteristic 0 is essentially equivalent to the study of higher traces on $A$ (cf. [3]) or on a differential graded algebra resolution of $A$ (cf. [6]). Quillen [11] represented cyclic cohomology classes of $A$ by means of certain traces on an algebra extension $A=R / I$ and showed that if the algebra $R$ is free, then any cyclic cohomology class can be realized as the class attached to a trace on this extension. Furthermore, Quillen obtained a description of cyclic homology as the inverse limit of a suitable functor on the category of extensions of the algebra $A$.

The computation of the cyclic homology groups of $A$, in the special case where $A=k \Pi$ is the group algebra of a group $\Pi$, is due to Burghelea [2] (see also Chapter 7 of Loday's book [8]). It turns out that the groups $H C_{*}(k \Pi)$ admit a decomposition, indexed by the conjugacy classes of $\Pi$, into the direct sum of certain components, which can themselves be expressed in terms of the homology groups of certain subquotients of $\Pi$. In particular, the homology groups of $\Pi$ appear as subgroups of $H C_{*}(k \Pi)$.

Using the above circle of ideas, we describe in this paper the homology groups of $\Pi$ as the inverse limit of suitable functors on the category of extensions $\Pi=G / H$. More precisely, our main result (Theorems 5.2 and 5.5) is that for all $n \geqslant 1$, there are isomorphisms

$$
H_{2 n}(\Pi, k) \simeq \lim _{\longleftarrow} \frac{\mathfrak{h}^{n}+(k H \cap[k G, k G])}{\mathfrak{h}^{n+1}+(k H \cap[k G, k G])}
$$

and

$$
H_{2 n+1}(\Pi, k) \simeq \lim _{\longleftarrow} \frac{\mathfrak{h}^{n+1} \cap\left[\mathfrak{h} \cdot k G, \mathfrak{h}^{n-1} \cdot k G\right]}{\mathfrak{h}^{n+1} \cap\left[\mathfrak{h} \cdot k G, \mathfrak{h}^{n} \cdot k G\right]}
$$

Research co-funded by European Social Fund and National Resources (EPEAEK II) PYTHAGORAS. Received July 3, 2007; published on February 26, 2008. 2000 Mathematics Subject Classification: 19D55, 16S34, $20 \mathrm{~J} 15$.

Key words and phrases: group rings, cyclic homology, group homology, group extensions.

This article is available at http://intlpress.com/HHA/v10/n1/a10

Copyright (C) 2008, International Press. Permission to copy for private use granted. 
where the inverse limits are over the category of extensions of the group $\Pi$. Here, for any extension $\Pi=G / H$, we denote by $\mathfrak{h}=\mathfrak{h}_{k}$ the augmentation ideal of the group algebra $k H$, whereas for any two subspaces $U, V \subseteq k G$ we denote by $[U, V]$ the $k$-linear span of the commutators $x y-y x, x \in U, y \in V$. In particular, since $k H \cap[k G, k G] \subseteq$ $\mathfrak{h}$ for any extension $\Pi=G / H$, there is an isomorphism

$$
H_{2}(\Pi, k) \simeq \lim _{\longleftarrow} \frac{\mathfrak{h}}{\mathfrak{h}^{2}+(k H \cap[k G, k G])} .
$$

In $[\mathbf{1 0}]$ it has been noted that, given a free presentation $\Pi=F / N$, the homology groups $H_{2 n}(\Pi, k)$ embed à la Gruenberg into suitable subquotients of the group algebra $k F$. Our analysis provides (Corollary 5.3) a similar embedding for the odd dimensional homology groups $H_{2 n+1}(\Pi, k)$.

We observe that Hopf's formula for the second integral homology of a group may be restated as the assertion that there is an isomorphism

$$
H_{2}(\Pi, \mathbb{Z}) \simeq \lim _{\longleftarrow} \frac{\mathfrak{h}_{\mathbb{Z}}}{\mathfrak{h}_{\mathbb{Z}}^{2}+(\mathbb{Z} H \cap[\mathbb{Z} G, \mathbb{Z} G])},
$$

where $\mathfrak{h}_{\mathbb{Z}}$ is the augmentation ideal of the group ring $\mathbb{Z} H$ for any extension $\Pi=G / H$. This indicates that some of the results obtained in this paper, under the assumption that $k$ is a field of characteristic 0 , may be valid over the ring $\mathbb{Z}$ as well. It may be mentioned here that Emmanouil and Mikhailov [5] have obtained a formula for the even dimensional homology of a group with coefficients in an arbitrary module as the inverse limit of a certain functor over the category of free presentations of the group. However the latter formula does not include our result for the even dimensional homology as a special case. It thus remains a challenging goal for the future to understand the relation between the methods of [5] and the formulae of the present paper, as well as their relation to the terms of the Gruenberg resolution [7]. Even though one cannot claim that our results provide an effective tool for computing group homology, we believe that (in the opposite direction) the evaluation of these inverse limits as group homology is intriguing and demands further investigation.

The contents of the paper are as follows: In Section 1, we record a few elementary observations relating the extensions of a group to those of the corresponding group algebra. In the subsequent section, we detail the behavior of Quillen's spectral sequences in the case where the extension of the group algebra is induced by a free presentation of the group. In Section 3, we examine the behavior of Connes' homomorphisms $\gamma$ with respect to the periodicity operator $S$ in cyclic homology. As a motivation for our main result, in Section 4, we reformulate Hopf's formula for $H_{2}$ by establishing the existence of an isomorphism as mentioned above. Finally, in the last section, we obtain our main result, describing the homology of a group as the inverse limit of a functor on the category of extensions of the group.

\section{Notation}

Let $k$ be a commutative ring.

(i) If $\varphi: G \longrightarrow H$ is a group homomorphism, then we denote by $\widetilde{\varphi}: k G \longrightarrow k H$ its $k$-linear extension to the respective group algebras. In other words, $\widetilde{\varphi}$ is the $k$ algebra homomorphism, which is defined by mapping any element $\sum_{g \in G} a_{g} g \in$ 
$k G$ onto the element $\sum_{g \in G} a_{g} \varphi(g) \in k H$.

(ii) Given a group $H$, we adopt Gruenberg's notation [7] and denote by $\mathfrak{h}$ the augmentation ideal of the group algebra $k H$. In the case where the dependence upon the ground ring $k$ is to be emphasized, we denote $\mathfrak{h}$ by $\mathfrak{h}_{k}$. If $H$ is a normal subgroup of a group $G$ and $n$ a non-negative integer, then we denote by $\mathfrak{h}^{n} \cdot k G$ the ideal of $k G$ generated by the ideal $\mathfrak{h}^{n} \subseteq k H$. We adopt an analogous notation for groups $H^{\prime}, H_{0}, N, \ldots$ and denote their augmentation ideals by $\mathfrak{h}^{\prime}, \mathfrak{h}_{0}, \mathfrak{n}, \ldots$ respectively.

(iii) If $R$ is a $k$-algebra, then for any two $k$-submodules $U, V \subseteq R$ we denote by $[U, V]$ the $k$-linear span of the commutators $x y-y x, x \in U, y \in V$.

\section{Group extensions and extensions of group algebras}

We fix a commutative ring $k$ and a group $\Pi$ and define the category $\mathfrak{E}_{g r p}(\Pi)$ of extensions of $\Pi$, as follows: The objects of $\mathfrak{E}_{g r p}(\Pi)$ are the short exact sequences of groups of the form

$$
1 \longrightarrow H \longrightarrow G \stackrel{\rho}{\longrightarrow} \Pi \longrightarrow 1
$$

and its morphisms are the commutative diagrams of the form

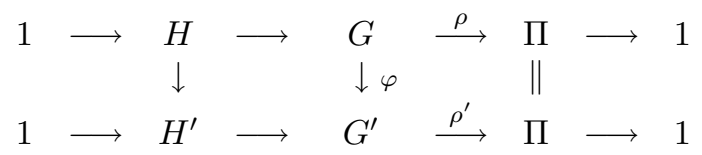

where $\varphi$ is a group homomorphism. In order to guarantee that the category $\mathfrak{E}_{g r p}(\Pi)$ be small, we require that any object of $\mathfrak{E}_{g r p}(\Pi)$ is such that the group $G$ therein can be obtained as a quotient of the free group on $c$ generators, where $c$ is some fixed cardinal number; for our purposes, we may choose $c=\max \left\{\aleph_{0}, \operatorname{card}(k), \operatorname{card}(\Pi)\right\}$.

In the same way, for any $k$-algebra $A$ we define the category $\mathfrak{E}_{a l g}(A)$ of algebra extensions of $A$, as follows: The objects of $\mathfrak{E}_{a l g}(A)$ are the short exact sequences

$$
0 \longrightarrow I \longrightarrow R \stackrel{p}{\longrightarrow} A \longrightarrow 0
$$

where $p$ is a $k$-algebra homomorphism, and its morphisms are the commutative diagrams of the form

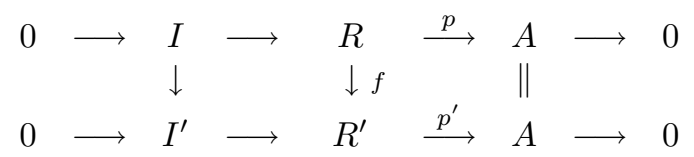

where $f$ is a $k$-algebra homomorphism. As before, in order to guarantee that the category $\mathfrak{E}_{a l g}(A)$ be small, we require that any object of $\mathfrak{E}_{a l g}(A)$ is such that the algebra $R$ therein can be obtained as a quotient of the free $k$-algebra on $c$ generators, where $c$ is some fixed cardinal number; for our purposes, we may choose $c=\max \left\{\aleph_{0}, \operatorname{card}(A)\right\}$.

We now consider the group algebra $k \Pi$ and define the functor $t: \mathfrak{E}_{g r p}(\Pi) \longrightarrow$ 
$\mathfrak{E}_{\text {alg }}(k \Pi)$, as follows: Any group extension (1) is mapped under $t$ onto the extension

$$
0 \longrightarrow \mathfrak{h} \cdot k G \longrightarrow k G \stackrel{\widetilde{\rho}}{\longrightarrow} k \Pi \longrightarrow 0
$$

of the group algebra $k \Pi$, whereas any morphism $(2)$ of $\mathfrak{E}_{g r p}(\Pi)$ is mapped onto the morphism of algebra extensions

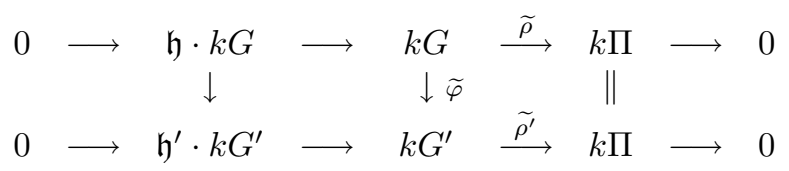

An extension

$$
0 \longrightarrow I \longrightarrow R \stackrel{p}{\longrightarrow} k \Pi \longrightarrow 0
$$

of the group algebra $k \Pi$ will be called admissible if there is at least one group extension (1), such that there is a morphism of algebra extensions

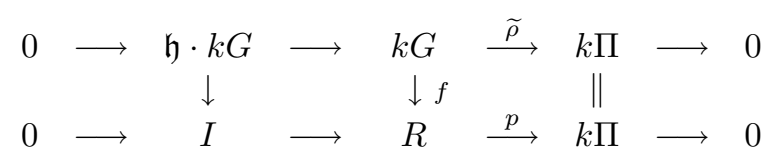

In what follows, we need the following property of admissible extensions.

Proposition 1.1. Consider an admissible extension (3) of the group algebra $k \Pi$. Then there is an extension

$$
1 \longrightarrow H_{0} \longrightarrow G_{0} \stackrel{\rho_{0}}{\longrightarrow} \Pi \longrightarrow 1
$$

of the group $\Pi$ and a morphism of algebra extensions

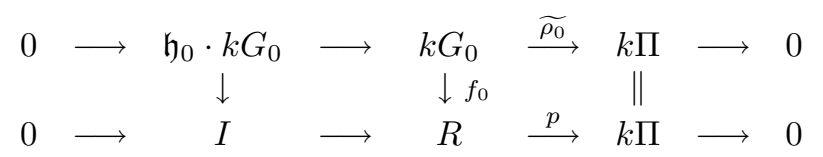

having the following property: For any extension (1) of the group $\Pi$ and any morphism (4) of algebra extensions, there exists a morphism of group extensions

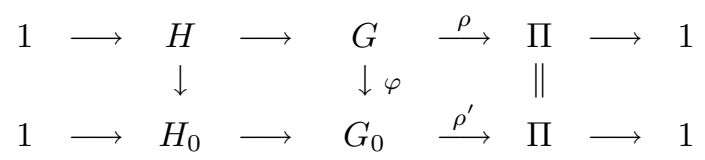

such that $f=f_{0} \circ \widetilde{\varphi}$.

Proof. Let $U(R) \subseteq R$ be the unit group of $R$ and define $G_{0}=\{r \in U(R): p(r) \in \Pi\}$. Then, the admissibility condition implies that $p\left(G_{0}\right)=\Pi$. (In fact, it is easily seen that the admissibility condition is equivalent to the equality $p\left(G_{0}\right)=\Pi$.) Let $\rho_{0}: G_{0} \longrightarrow$ $\Pi$ be the group homomorphism obtained by restricting $p$ to $G_{0}$ and consider the kernel $H_{0}=\operatorname{ker} \rho_{0}$. We define the $k$-algebra homomorphism $f_{0}: k G_{0} \longrightarrow R$ using the embedding of $G_{0}$ into the group of units $U(R) \subseteq R$, obtaining thereby a morphism of algebra extensions as in (5). If we are given a morphism of algebra extensions as in (4), then $f$ maps $G \subseteq k G$ into $G_{0}$ and hence we may consider the group homomorphism 
$\varphi: G \longrightarrow G_{0}$, which is defined by restricting $f$ to $G$. In this way, we obtain a morphism of group extensions as in (6), for which we obviously have $f=f_{0} \circ \widetilde{\varphi}$.

Remark 1.2. Using the language of category theory (cf. $[\mathbf{9}])$, an object $p$ of $\mathfrak{E}_{a l g}(k \Pi)$ is admissible if and only if the comma category $(t \downarrow p)$ of $t$-objects over $p$ is non-empty. If $p$ is such an object, then Proposition 1.1 can be expressed by saying that $(t \downarrow p)$ has a quasi-terminal object, i.e. an object $f_{0}$ such that $\operatorname{Hom}_{(t \downarrow p)}\left(f, f_{0}\right) \neq \emptyset$ for any object $f$ of $(t \downarrow p)$.

Proposition 1.1 will be used in the form of the following corollary.

Corollary 1.3. Let $\mathfrak{F}$ be a functor from the category $\mathfrak{E}_{\text {alg }}(k \Pi)$ of algebra extensions of $k \Pi$ to the category of $k$-modules. We consider an extension (1) of the group $\Pi$, the $k$-module $V$ obtained by applying the composition $\mathfrak{F} \circ t$ to it and assume that $\xi \in V$ is an element having the following property: For any parallel pair of morphisms in $\mathfrak{E}_{g r p}(\Pi)$

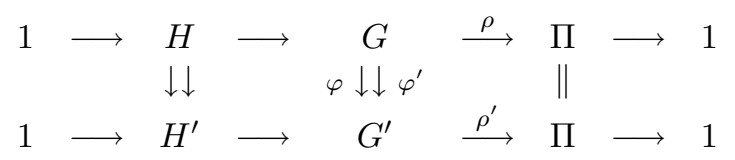

we have $(\mathfrak{F} \circ t)(\varphi)(\xi)=(\mathfrak{F} \circ t)\left(\varphi^{\prime}\right)(\xi) \in V^{\prime}$, where $V^{\prime}$ is the $k$-module obtained by applying the functor $\mathfrak{F} \circ t$ to the extension of $\Pi$ at the bottom of the above diagram. Then, for any parallel pair of morphisms in $\mathfrak{E}_{\text {alg }}(k \Pi)$

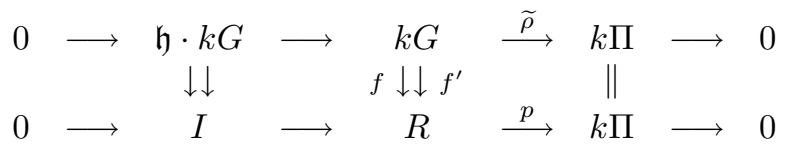

we have $\mathfrak{F}(f)(\xi)=\mathfrak{F}\left(f^{\prime}\right)(\xi) \in W$, where $W$ is the $k$-module obtained by applying the functor $\mathfrak{F}$ to the extension of $k \Pi$ at the bottom of the above diagram.

Proof. Let $\left(f, f^{\prime}\right)$ be a parallel pair of morphisms as in the statement. Then, the extension

$$
0 \longrightarrow I \longrightarrow R \stackrel{p}{\longrightarrow} k \Pi \longrightarrow 0
$$

is admissible. If

$$
1 \longrightarrow H_{0} \longrightarrow G_{0} \stackrel{\rho_{0}}{\longrightarrow} \Pi \longrightarrow 1
$$

and

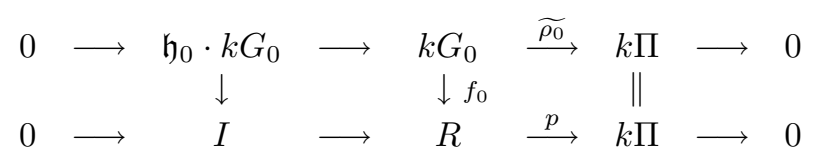

are as in the statement of Proposition 1.1, then we have $f=f_{0} \circ \widetilde{\varphi}$ and $f^{\prime}=f_{0} \circ \widetilde{\varphi}^{\prime}$ for a suitable parallel pair $\left(\varphi, \varphi^{\prime}\right)$ of morphisms in $\mathfrak{E}_{g r p}(\Pi)$. It follows that

$$
\mathfrak{F}(f)=\mathfrak{F}\left(f_{0} \circ \widetilde{\varphi}\right)=\mathfrak{F}\left(f_{0}\right) \circ \mathfrak{F}(\widetilde{\varphi})=\mathfrak{F}\left(f_{0}\right) \circ(\mathfrak{F} \circ t)(\varphi)
$$

and, similarly, $\mathfrak{F}\left(f^{\prime}\right)=\mathfrak{F}\left(f_{0}\right) \circ(\mathfrak{F} \circ t)\left(\varphi^{\prime}\right)$. Since we have $(\mathfrak{F} \circ t)(\varphi)(\xi)=(\mathfrak{F} \circ t)\left(\varphi^{\prime}\right)(\xi)$, in view of our assumption on $\xi$, we may conclude that $\mathfrak{F}(f)(\xi)=\mathfrak{F}\left(f^{\prime}\right)(\xi)$. 


\section{Quillen's spectral sequences}

We fix a field $k$ of characteristic 0 . Let $\Pi$ be a group and consider an algebra extension of the group algebra $k \Pi$. Quillen has constructed in [11] two spectral sequences, which converge to the (reduced) cyclic homology of $k \Pi$, with $E^{1}$-terms described by means of the given extension, and studied them carefully in the special case of a free algebra presentation. We are interested in the case where the algebra extension is induced by a free presentation of the group $\Pi$. More precisely, let $F$ be a free group which maps surjectively onto $\Pi$ and consider the kernel $N$ of the homomorphism $F \longrightarrow \Pi$ and the associated algebra extension

$$
0 \longrightarrow I \longrightarrow k F \stackrel{p}{\longrightarrow} k \Pi \longrightarrow 0
$$

where $I=\mathfrak{n} \cdot k F$. In the terminology of [4], the group algebra $R=k F$ is quasi-free, i.e. the $R$-bimodule of non-commutative differential 1-forms

$$
\Omega_{R}^{1}=\operatorname{ker}(R \otimes R \stackrel{m}{\longrightarrow} R)
$$

is projective. (Here, $m$ denotes the multiplication of $R$.) The ideal $I$ is known to be free (and hence flat) both as a left and right $R$-module (cf. [7]) and the Hochschild homology groups $H_{n}\left(R,{ }_{-}\right)$vanish for all $n \geqslant 2$. In view of the flatness of $I$, the cyclic group $\mathbb{Z} / n \mathbb{Z}=\left\langle\sigma_{n}>\right.$ acts naturally on the Hochschild homology groups $H_{*}\left(R, I^{n}\right)$ and $H_{*}\left(R, R / I^{n}\right)$, in such a way that the exact sequence

$$
\begin{aligned}
0 \rightarrow H_{1}\left(R, I^{n}\right) \rightarrow H_{1}(R, R) \rightarrow H_{1}\left(R, R / I^{n}\right) & \rightarrow H_{0}\left(R, I^{n}\right) \\
& \rightarrow H_{0}(R, R) \rightarrow H_{0}\left(R, R / I^{n}\right) \rightarrow 0
\end{aligned}
$$

is $\mathbb{Z} / n \mathbb{Z}$-equivariant, where $\sigma_{n}$ acts trivially on $H_{*}(R, R)$ (cf. [11, Prop, 2.16]). It follows that $\sigma_{n}$ acts trivially on $H_{1}\left(R, I^{n}\right)$ and $H_{0}\left(R, R / I^{n}\right)$.

Using Burghelea's computation [2] of the cyclic homology of group algebras, the fact that the centralizer of any non-trivial element of $F$ is infinite cyclic and the vanishing of the homology groups $H_{n}(F, k)$ for all $n \geqslant 2$, we obtain the following result.

Proposition 2.1. The cyclic homology groups of the free group algebra $R=k F$ are given by

$$
H C_{n}(R)= \begin{cases}H_{0}(F, k) & \text { if } n>0 \text { is even } \\ H_{1}(F, k) & \text { if } n \geqslant 1 \text { is odd. }\end{cases}
$$

In particular, the reduced cyclic homology $\bar{H} C_{2 n}(R)$ of $R$ vanishes if $n>0$.

\subsection{The odd Connes homomorphisms}

Letting $R=k F$ and $I=\mathfrak{n} \cdot k F$ as above, the first of Quillen's spectral sequences has $E^{1}$-term (located in the first quadrant) given by:

$$
E_{p q}^{1}=\left\{\begin{array}{cc}
\bar{H} C_{q}(R) & \text { if } p=0 \\
H_{q-p+1}\left(R, I^{p}\right)_{\sigma} & \text { if } p \geqslant 1
\end{array} \Longrightarrow \bar{H} C_{n}(k \Pi) .\right.
$$

Here, $H_{q-p+1}\left(R, I^{p}\right)_{\sigma}$ denotes the coinvariance of the Hochschild homology group $H_{q-p+1}\left(R, I^{p}\right)$ under the action of the cyclic group $\left\langle\sigma_{p}\right\rangle$ of order $p$. It follows that the $E^{1}$-term of the spectral sequence is concentrated on the $p=0$ axis and the lines 
$q=p$ and $q=p-1$. Moreover, we have $E_{02 n}^{1}=0$ for all $n>0$. It follows that, besides the differentials

$$
d^{1}: E_{n+1 n}^{1} \longrightarrow E_{n n}^{1}, n \geqslant 0,
$$

the only possibly non-zero differentials of the spectral sequence are

$$
d^{1}: E_{11}^{1} \longrightarrow E_{01}^{1}
$$

and the higher order differentials

$$
E_{n+1 n+1}^{2}=E_{n+1 n+1}^{n+1} \stackrel{d^{n+1}}{\longrightarrow} E_{02 n+1}^{n+1}=E_{02 n+1}^{1}, \quad n \geqslant 1 .
$$

We conclude that there is an exact sequence

$$
E_{02 n+1}^{1} \stackrel{\text { edge }}{\longrightarrow} H C_{2 n+1}(k \Pi) \stackrel{\text { edge }}{\longrightarrow} E_{n+1 n}^{1} \stackrel{d^{1}}{\longrightarrow} E_{n n}^{1}
$$

for all $n \geqslant 0$. The edge homomorphism

$$
H C_{2 n+1}(k \Pi) \longrightarrow E_{n+1 n}^{1}=I^{n+1} /\left[I, I^{n}\right]
$$

is, by definition, the odd Connes homomorphism $\gamma_{2 n+1}$ (cf. [3, I, Prop. 4] and [11]). Identifying the kernel of the differential $d^{1}$ as in [11, Prop. 4.9], we obtain the following result.

Theorem 2.2. (cf. [11, Thm. 5.11]) Let $F$ be a free group mapping surjectively onto $\Pi$ and consider the kernel $I$ of the associated algebra homomorphism $p: k F \longrightarrow k \Pi$.

(i) For all $n \geqslant 1$, there is an exact sequence

$$
H C_{2 n+1}(k F) \stackrel{p_{*}}{\longrightarrow} H C_{2 n+1}(k \Pi) \stackrel{\gamma_{2 n+1}}{\longrightarrow} I^{n+1} /\left[I, I^{n}\right] \stackrel{d^{1}}{\longrightarrow} H_{1}\left(k F, I^{n}\right),
$$

where $\gamma_{2 n+1}$ is Connes' homomorphism. The kernel of $d^{1}: I^{n+1} /\left[I, I^{n}\right] \longrightarrow$ $H_{1}\left(k F, I^{n}\right)$ coincides with the kernel of the map $\delta: I^{n+1} /\left[I, I^{n}\right] \longrightarrow$ $\Omega_{k F}^{1} /\left[k F, \Omega_{k F}^{1}\right]$ induced by the de Rham differential $d: k F \longrightarrow \Omega_{k F}^{1}$.

(ii) There is an exact sequence

$$
H C_{1}(k F) \stackrel{p_{*}}{\longrightarrow} H C_{1}(k \Pi) \stackrel{\gamma_{1}}{\longrightarrow} I /[I, k F] \longrightarrow k F /[k F, k F],
$$

where $\gamma_{1}$ is Connes' homomorphism and the unlabelled arrow is induced by the inclusion $I \hookrightarrow k F$.

Remark 2.3. Let $F, p: k F \longrightarrow k \Pi$ and $I=\operatorname{ker} p$ be as above.

(i) We consider the semi-direct product $k F \oplus \Omega_{k F}^{1}$ of $k F$ by the $k F$-bimodule $\Omega_{k F}^{1}$ (which is an ideal of square zero in $k F \oplus \Omega_{k F}^{1}$ ). The algebra homomorphism $p^{\prime}: k F \oplus \Omega_{k F}^{1} \longrightarrow k \Pi$, which is defined as the projection onto $k F$ followed by $p$, has kernel $J=I \oplus \Omega_{k F}^{1}$. Let

$$
u, v: k F \longrightarrow k F \oplus \Omega_{k F}^{1}
$$

be the algebra homomorphisms, which are defined by letting $u(x)=(x, 0)$ and $v(x)=(x, d x)$ for all $x \in k F$. (Here, we denote by $d: k F \longrightarrow \Omega_{k F}^{1}$ the de Rham differential.) Since both $u$ and $v$ lift the identity of $k \Pi$, there are induced $k$-linear 
maps

$$
u_{*}, v_{*}: I^{n+1} /\left[I, I^{n}\right] \longrightarrow J^{n+1} /\left[J, J^{n}\right]
$$

for all $n \geqslant 1$, which have the following property: If $a \in I^{n+1} /\left[I, I^{n}\right]$ is an element such that $u_{*}(a)=v_{*}(a) \in J^{n+1} /\left[J, J^{n}\right]$, then $a=\gamma_{2 n+1}(b)$ for a suitable element $b \in H C_{2 n+1}(k \Pi)$, where $\gamma_{2 n+1}: H C_{2 n+1}(k \Pi) \longrightarrow I^{n+1} /\left[I, I^{n}\right]$ is the odd Connes homomorphism. This follows from the exact sequence of Theorem 2.2(i), by repeating the argument used in the proof of [11, Thm. 5.17].

(ii) The exact sequences of Theorem 2.2 provide us with an answer to the question raised in [10, bottom of p. 995]. Indeed, it follows that the kernel of the odd Connes homomorphism

$$
\gamma_{2 n+1}: H C_{2 n+1}(k \Pi) \longrightarrow I^{n+1} /\left[I, I^{n}\right]
$$

coincides with the image of the induced map

$$
p_{*}: H C_{2 n+1}(k F) \longrightarrow H C_{2 n+1}(k \Pi)
$$

for all $n \geqslant 0$. On the other hand, the image of the latter map is easily seen to be equal to

$$
\begin{aligned}
H_{1}(\Pi, k) \subseteq H_{2 n+1}(\Pi, k) \oplus \cdots \oplus H_{3}(\Pi, k) \oplus H_{1}(\Pi, k) & \\
& =H C_{2 n+1}(k \Pi)_{[1]} \subseteq H C_{2 n+1}(k \Pi) .
\end{aligned}
$$

Here, we denote by $H C_{2 n+1}(k \Pi)_{[1]}$ the component corresponding to the conjugacy class of 1 in the Burghelea decomposition of $H C_{2 n+1}(k \Pi)$ (cf. [2]).

\subsection{The even Connes homomorphisms}

As far as the second of the spectral sequences that Quillen constructed in [11] is concerned, we note that the assumption that the presenting algebra is free can be replaced by the assumption that it be quasi-free without any change in the analysis therein. In particular, letting $R=k F$, where $F$ is a free group mapping onto $\Pi$, and denoting by $I$ the kernel of the associated algebra homomorphism $k F \longrightarrow k \Pi$, the $E^{1}$-term of the spectral sequence (located in the first quadrant) is given by

$$
E_{p q}^{1}=H_{q-p}\left(R, R / I^{p+1}\right)_{\sigma} \Longrightarrow H C_{n}(k \Pi) .
$$

Here, $H_{q-p}\left(R, R / I^{p+1}\right)_{\sigma}$ denotes the coinvariance of $H_{q-p}\left(R, R / I^{p+1}\right)$ under the action of the cyclic group $<\sigma_{p+1}>$ of order $p+1$. Since the $E^{1}$-term is concentrated on the lines $q=p$ and $q=p+1$, we obtain the following result, which is a special case of $[\mathbf{1 1}$, Thm. 5.13].

Theorem 2.4. Let $F$ be a free group mapping surjectively onto $\Pi$ and consider the kernel $I$ of the associated algebra homomorphism $p: k F \longrightarrow k \Pi$. Then, for any $n \geqslant 0$ there is an exact sequence

$$
\begin{aligned}
0 \longrightarrow H C_{2 n}(k \Pi) \stackrel{\gamma_{2 n}}{\longrightarrow} k F /\left([k F, k F]+I^{n+1}\right) \stackrel{d^{1}}{\longrightarrow} \\
H_{1}\left(k F, k F / I^{n}\right)_{\sigma} \longrightarrow H C_{2 n-1}(k \Pi) \longrightarrow 0,
\end{aligned}
$$

where $\gamma_{2 n}$ is (by definition) the even Connes homomorphism. The kernel of

$$
d^{1}: k F /\left([k F, k F]+I^{n+1}\right) \longrightarrow H_{1}\left(k F, k F / I^{n}\right)_{\sigma}
$$


coincides with the kernel of the map

$$
\delta: k F /\left([k F, k F]+I^{n+1}\right) \longrightarrow \Omega_{k F}^{1} /\left(\left[k F, \Omega_{k F}^{1}\right]+I^{n} \Omega_{k F}^{1}\right),
$$

which is induced by the de Rham differential $d: k F \longrightarrow \Omega_{k F}^{1}$.

Remark 2.5. Let $F, p: k F \longrightarrow k \Pi$ and $I=\operatorname{ker} p$ be as above. We also consider the semi-direct product $R^{\prime}=k F \oplus \Omega_{k F}^{1}$ and the algebra homomorphism $p^{\prime}: R^{\prime} \longrightarrow k \Pi$ with kernel $J$, defined in Remark 2.3(i). Since the homomorphisms $u, v$ defined in loc. cit. lift the identity of $k \Pi$, there are induced $k$-linear maps

$$
u_{*}, v_{*}: k F /\left([k F, k F]+I^{n+1}\right) \longrightarrow R^{\prime} /\left(\left[R^{\prime}, R^{\prime}\right]+J^{n+1}\right)
$$

for all $n \geqslant 0$, which have the following property: If $a \in k F /\left([k F, k F]+I^{n+1}\right)$ is an element such that $u_{*}(a)=v_{*}(a) \in R^{\prime} /\left(\left[R^{\prime}, R^{\prime}\right]+J^{n+1}\right)$, then $a=\gamma_{2 n}(b)$ for a suitable element $b \in H C_{2 n}(k \Pi)$, where $\gamma_{2 n}: H C_{2 n}(k \Pi) \longrightarrow k F /\left([k F, k F]+I^{n+1}\right)$ is the even Connes homomorphism. This follows from the exact sequence of Theorem 2.4, by repeating the argument used in the proof of [11, Thm. 5.18].

\section{Connes homomorphisms and the operator $S$}

Let $A$ be an algebra over a field $k$ of characteristic 0 and fix an extension

$$
0 \longrightarrow I \longrightarrow R \longrightarrow A \longrightarrow 0 \text {. }
$$

Our goal in this section is to make explicit the relation between the operator $S$ in the cyclic homology of $A$ and Connes homomorphisms associated with the given extension.

\subsection{The odd case}

For any linear map $\tau: I^{m+1} \longrightarrow V$, which vanishes on $\left[R, I^{m+1}\right]$, and all $n \geqslant m$ there is a cyclic cohomology class $\operatorname{cn}_{2 n+1}(\tau) \in H C^{2 n+1}(A, V)$, which is induced by the Chern character form associated with $\tau$ and any linear lifting of the projection $R \longrightarrow A$ (cf. [11, Part II, $\S 1.2]$ ). The following properties are immediate consequences of the definitions:

- Let $f: V \longrightarrow V^{\prime}$ be a linear map and consider the induced map

$$
f_{*}: H C^{2 n+1}(A, V) \longrightarrow H C^{2 n+1}\left(A, V^{\prime}\right) .
$$

Then we have $f_{*}\left(\mathrm{cn}_{2 n+1}(\tau)\right)=\mathrm{cn}_{2 n+1}(f \circ \tau)$ for all $n \geqslant m$.

- Let $m^{\prime} \geqslant m$ and consider the restriction $\tau^{\prime}: I^{m^{\prime}+1} \longrightarrow V$ of $\tau$ on $I^{m^{\prime}+1}$. Then we have $\operatorname{cn}_{2 n+1}(\tau)=\operatorname{cn}_{2 n+1}\left(\tau^{\prime}\right)$ for all $n \geqslant m^{\prime}$.

As shown in [11, Thm. 3], the odd Connes homomorphism

$$
\gamma_{2 n+1}: H C_{2 n+1}(A) \longrightarrow I^{n+1} /\left[I, I^{n}\right]
$$

is the linear map associated with the cyclic cohomology class $(n+1) ! \mathrm{cn}_{2 n+1}\left(\tau_{2 n+1}\right)$, where

$$
\tau_{2 n+1}: I^{n+1} \longrightarrow I^{n+1} /\left[I, I^{n}\right]
$$

is the canonical projection. 
Lemma 3.1. The following diagram is commutative for all $n \geqslant 1$

$$
\begin{array}{ccc}
H C_{2 n+1}(A) & \stackrel{\gamma_{2 n+1}}{\longrightarrow} & I^{n+1} /\left[I, I^{n}\right] \\
(n+1) S \downarrow & & \downarrow \\
H C_{2 n-1}(A) & \stackrel{\gamma_{2 n-1}}{\longrightarrow} & I^{n} /\left[I, I^{n-1}\right] .
\end{array}
$$

Here, $S$ is the periodicity operator in cyclic homology and the unlabelled vertical arrow is induced by the inclusion $I^{n+1} \hookrightarrow I^{n}$.

Proof. In view of the discussion above, the composition

$$
H C_{2 n+1}(A) \stackrel{\gamma_{2 n+1}}{\longrightarrow} I^{n+1} /\left[I, I^{n}\right] \longrightarrow I^{n} /\left[I, I^{n-1}\right]
$$

is associated with the element $(n+1) ! \mathrm{cn}_{2 n+1}\left(\tau_{2 n+1}^{\prime}\right) \in H C^{2 n+1}\left(A, I^{n} /\left[I, I^{n-1}\right]\right)$, where $\tau_{2 n+1}^{\prime}$ is the composition

$$
I^{n+1} \stackrel{\tau_{2 n+1}}{\longrightarrow} I^{n+1} /\left[I, I^{n}\right] \longrightarrow I^{n} /\left[I, I^{n-1}\right] .
$$

On the other hand, the composition

$$
H C_{2 n+1}(A) \stackrel{(n+1) S}{\longrightarrow} H C_{2 n-1}(A) \stackrel{\gamma_{2 n-1}}{\longrightarrow} I^{n} /\left[I, I^{n-1}\right]
$$

is associated with the element $(n+1) S^{\prime}\left(n ! \mathrm{cn}_{2 n-1}\left(\tau_{2 n-1}\right)\right) \in H C^{2 n+1}\left(A, I^{n} /\left[I, I^{n-1}\right]\right)$, where $S^{\prime}$ denotes the periodicity operator in cyclic cohomology. In view of $[\mathbf{1 1}$, Thm. 1], we have

$$
S^{\prime}\left(\operatorname{cn}_{2 n-1}\left(\tau_{2 n-1}\right)\right)=\operatorname{cn}_{2 n+1}\left(\tau_{2 n-1}\right) \in H C^{2 n+1}\left(A, I^{n} /\left[I, I^{n-1}\right]\right) .
$$

The result follows, since $\tau_{2 n+1}^{\prime}$ is the restriction of $\tau_{2 n-1}: I^{n} \longrightarrow I^{n} /\left[I, I^{n-1}\right]$ to $I^{n+1}$.

\subsection{The even case}

For any linear map $\tau: R \longrightarrow V$, which vanishes on $[R, R]+I^{n+1}$, there is a cyclic cohomology class $\mathrm{cn}_{2 n}(\tau) \in H C^{2 n}(A, V)$, which is induced by the Chern-Simons form associated with $\tau$ and any linear lifting of the projection $R \longrightarrow A$ (cf. [11, Part II, $\S 1.2])$. Let $f: V \longrightarrow V^{\prime}$ be a linear map and consider the induced map

$$
f_{*}: H C^{2 n}(A, V) \longrightarrow H C^{2 n}\left(A, V^{\prime}\right) .
$$

Then, as in the odd case, we have $f_{*}\left(\mathrm{cn}_{2 n}(\tau)\right)=\mathrm{cn}_{2 n}(f \circ \tau)$. As shown in [11, Thm. 3], the even Connes homomorphism

$$
\gamma_{2 n}: H C_{2 n}(A) \longrightarrow R /\left([R, R]+I^{n+1}\right)
$$

is associated with the element $(n+1) ! \mathrm{cn}_{2 n}\left(\tau_{2 n}\right) \in H C^{2 n}\left(A, R /\left([R, R]+I^{n+1}\right)\right)$, where

$$
\tau_{2 n}: R \longrightarrow R /\left([R, R]+I^{n+1}\right)
$$

is the canonical projection. 
Lemma 3.2. The following diagram is commutative for all $n \geqslant 1$

$$
\begin{array}{ccc}
H C_{2 n}(A) & \stackrel{\gamma_{2 n}}{\longrightarrow} & R /\left([R, R]+I^{n+1}\right) \\
(n+1) S \downarrow & & \downarrow \\
H C_{2 n-2}(A) & \stackrel{\gamma_{2 n-2}}{\longrightarrow} & R /\left([R, R]+I^{n}\right) .
\end{array}
$$

Here, we denote by $S$ the periodicity operator in cyclic homology, while the unlabelled vertical arrow is induced by the identity map of $R$.

Proof. In view of the discussion above, the composition

$$
H C_{2 n}(A) \stackrel{\gamma_{2 n}}{\longrightarrow} R /\left([R, R]+I^{n+1}\right) \longrightarrow R /\left([R, R]+I^{n}\right)
$$

is associated with the element $(n+1) ! \mathrm{cn}_{2 n}\left(\tau_{2 n-2}\right) \in H C^{2 n}\left(A, R /\left([R, R]+I^{n}\right)\right)$. On the other hand, the composition

$$
H C_{2 n}(A) \stackrel{(n+1) S}{\longrightarrow} H C_{2 n-2}(A) \stackrel{\gamma_{2 n-2}}{\longrightarrow} R /\left([R, R]+I^{n}\right)
$$

is associated with the element $(n+1) S^{\prime}\left(n ! \mathrm{cn}_{2 n-2}\left(\tau_{2 n-2}\right)\right) \in H C^{2 n}(A, R /([R, R]+$ $\left.I^{n}\right)$ ), where $S^{\prime}$ denotes the periodicity operator in cyclic cohomology. The result follows since

$$
S^{\prime}\left(\mathrm{cn}_{2 n-2}\left(\tau_{2 n-2}\right)\right)=\mathrm{cn}_{2 n}\left(\tau_{2 n-2}\right) \in H C^{2 n}\left(A, R /\left([R, R]+I^{n}\right)\right)
$$

(cf. $[\mathbf{1 1}$, Thm. 1]).

\section{Extensions and $\boldsymbol{H}_{2}(-, \mathbb{Z})$}

As a motivation for our main result, which is to be presented in the next section, we describe the second integral homology of a group as the inverse limit of a certain functor on the category of extensions of the group.

\subsection{Some generalities on inverse limits}

Let $C$ be a small category, $\mathfrak{A}$ the category of modules over a ring $k$ and $\mathfrak{F}: C \longrightarrow \mathfrak{A}$ a functor. Then, the inverse $\operatorname{limit} \lim \mathfrak{F}$ of $\mathfrak{F}$ is the $k$-module consisting of all families $\left(x_{c}\right)_{c} \in \prod_{c \in C} \mathfrak{F}(c)$, which are compatible in the following sense: For any two objects $c, c^{\prime} \in C$ and any morphism $a \in \operatorname{Hom}_{C}\left(c, c^{\prime}\right)$, we have $\mathfrak{F}(a)\left(x_{c}\right)=x_{c^{\prime}} \in \mathfrak{F}\left(c^{\prime}\right)$.

Assume that $C$ has an initial object $c_{0}$; then the set $\operatorname{Hom}_{C}\left(c_{0}, c\right)$ is a singleton for any object $c \in C$ (cf. $[\mathbf{9}, \mathrm{Ch} . \mathrm{I}, \S 5])$. In that case, it is easily seen that the linear map

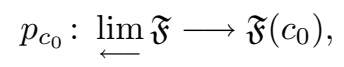

which is given by letting $\left(x_{c}\right)_{c} \mapsto x_{c_{0}}$ for any $\left(x_{c}\right)_{c} \in \lim \mathfrak{F}$, is an isomorphism. We say that an object $c_{0}$ of $C$ is quasi-initial if the set $\operatorname{Hom}_{C}\left(c_{0}, c\right)$ is non-empty for any object $c \in C$.

Example 4.1. Let $\Pi$ be a group and consider the category of extensions $\mathfrak{E}_{g r p}(\Pi)$ of $\Pi$. Then, any free presentation

$$
1 \longrightarrow N \longrightarrow F \stackrel{\sigma}{\longrightarrow} \Pi \longrightarrow 1
$$

of $\Pi$ is easily seen to be a quasi-initial object of the category $\mathfrak{E}_{g r p}(\Pi)$. 
Lemma 4.2. Assume that $C$ has a quasi-initial object $c_{0}$. Then for any functor $\mathfrak{F}: C \longrightarrow \mathfrak{A}$ the linear map (7) is injective, whereas its image consists of those elements $x \in \mathfrak{F}\left(c_{0}\right)$ which are such that for any object $c \in C$ and any morphisms $a, b \in$ $\operatorname{Hom}_{C}\left(c_{0}, c\right)$ we have $\mathfrak{F}(a)(x)=\mathfrak{F}(b)(x) \in \mathfrak{F}(c)$.

Proof. The proof is straightforward.

Let $\mathfrak{F}, \mathfrak{G}$ be two functors from $C$ to $\mathfrak{A}$. Then a natural transformation $\eta: \mathfrak{F} \longrightarrow \mathfrak{G}$ induces a linear map

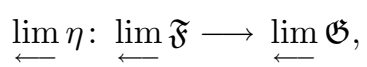

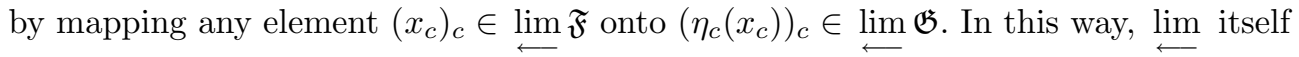
becomes a functor from the functor category $\mathfrak{A}^{C}$ to $\mathfrak{A}$.

Lemma 4.3. The inverse limit functor $\lim : \mathfrak{A}^{C} \longrightarrow \mathfrak{A}$ is left exact.

Proof. Since the kernel of a linear map is easily seen to be the limit of a certain functor from the category $\bullet \Longleftrightarrow \bullet$ to $\mathfrak{A}$ (cf. $[\mathbf{9}$, Ch. VIII, $\S 1]$ ), the result follows from the fact that limits commute with each other (cf. [9, Ch. IX, § 8]).

\subsection{A reformulation of Hopf's formula}

Let $\Pi$ be a group and consider the category $\mathfrak{E}_{g r p}(\Pi)$ of extensions of $\Pi$. We define the functor

$$
\Lambda: \mathfrak{E}_{g r p}(\Pi) \longrightarrow A b,
$$

where $A b$ is the category of abelian groups, as follows: An extension (1) of $\Pi$ is mapped onto the kernel $H /[H, G]$ of the associated central extension

$$
1 \longrightarrow H /[H, G] \longrightarrow G /[H, G] \stackrel{\bar{\rho}}{\longrightarrow} \Pi \longrightarrow 1,
$$

while a morphism of extensions (2) is mapped onto the induced additive map between the kernels of the associated central extensions.

Proposition 4.4. There is an isomorphism $\lim _{\longleftarrow} \Lambda \simeq H_{2}(\Pi, \mathbb{Z})$.

Proof. Let us fix a free presentation (8) of the group П. Then, in view of Example 4.1 and Lemma 4.2, the natural map $p: \lim \Lambda \longrightarrow N /[N, F]$ is injective. Therefore, the result will follow from Hopf's formula (cf. [1, Ch. II, Thm. 5.3]), provided that we establish the equality $\operatorname{im} p=(N \cap[F, F]) /[N, F]$.

Proof of the inclusion $\subseteq$ : Let us consider an element $\xi \in \operatorname{im} p$. We have to show that $\xi$ is contained in the kernel of the group homomorphism $N /[N, F] \longrightarrow F /[F, F]$, which is induced by the inclusion $N \hookrightarrow F$. We note that $F /[F, F]$ is the abelian group obtained 
by applying the functor $\Lambda$ to the trivial extension

$$
1 \longrightarrow F \longrightarrow F \times \Pi \longrightarrow \Pi \longrightarrow 1 \text {. }
$$

We also consider the morphisms of extensions

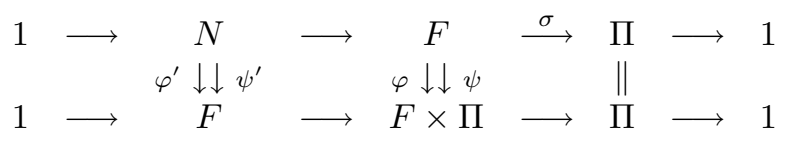

where $\varphi(x)=(x, \sigma(x))$ and $\psi(x)=(1, \sigma(x))$ for any element $x \in F$. It is clear that the map $\varphi^{\prime}: N \longrightarrow F$ is the inclusion, whereas $\psi^{\prime}: N \longrightarrow F$ is trivial. On the other hand, being a component of an element of $\lim \Lambda$, the element $\xi$ is equalized by the parallel pair of maps

$$
\overline{\varphi^{\prime}}, \overline{\psi^{\prime}}: N /[N, F] \longrightarrow F /[F, F] .
$$

We conclude that $\xi$ is indeed contained in the kernel of the map $N /[N, F] \longrightarrow$ $F /[F, F]$, which is induced by the inclusion of $N$ into $F$.

Proof of the inclusion $\supseteq$ : Let $x \in N \cap[F, F]$ and consider the class $\bar{x} \in$ $(N \cap[F, F]) /[N, F]$. For any extension (1), we fix a morphism of extensions

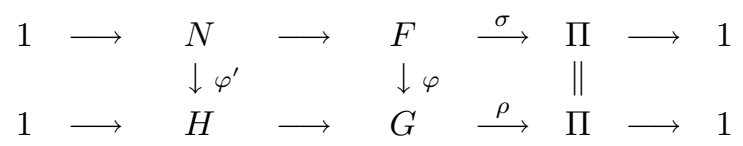

and consider the induced morphism between the associated central extensions

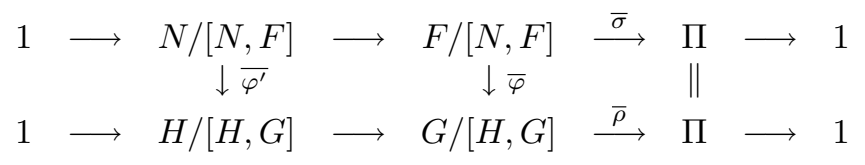

We claim that the residue class $\overline{\varphi^{\prime}}(\bar{x})$ of $\varphi^{\prime}(x) \in H$ in the quotient $H /[H, G]$ does not depend upon the choice of the homomorphism $\varphi: F \longrightarrow G$, which lifts the identity of $\Pi$. Indeed, let $\psi: F \longrightarrow G$ be another homomorphism lifting the identity of $\Pi$ and consider the induced homomorphism $\bar{\psi}: F /[N, F] \longrightarrow G /[H, G]$. Since both $\bar{\varphi}$ and $\bar{\psi}$ lift the identity of $\Pi$, it is easily seen that there is a (unique) group homomorphism

$$
\theta: F /[N, F] \longrightarrow H /[H, G]
$$

such that $\bar{\psi}(\omega)=\bar{\varphi}(\omega) \theta(\omega)$ for all $\omega \in F /[N, F]$. Since $x \in[F, F]$ and the group $H /[H, G]$ is abelian, we conclude that $\theta(\bar{x})$ is trivial and hence $\bar{\psi}(\bar{x})=\bar{\varphi}(\bar{x}) \in$ $G /[H, G]$, as needed.

The assignment of the element $\overline{\varphi^{\prime}}(\bar{x}) \in H /[H, G]$ to the extension (1) defines a family, which is easily seen to be compatible. (This is an immediate consequence of the already established independence of the element $\overline{\varphi^{\prime}}(\bar{x})$ upon the choice of $\varphi$.) Let $\mathfrak{X}$ be the element of the inverse $\operatorname{limit} \lim \Lambda$ defined in this way. Considering the identity map of $F$, it follows that the component of $\mathfrak{X}$ corresponding to the free presentation (8) is precisely the residue class $\bar{x} \in N /[N, F]$ of $x \in N$. Therefore, $\bar{x}=p(\mathfrak{X}) \in \operatorname{im} p$. 
Remark 4.5. Let $\Pi$ be a group.

(i) In view of [10, Prop. 1], the functor $\Lambda$ defined above is naturally isomorphic to the functor $\Lambda^{\prime}: \mathfrak{E}_{g r p}(\Pi) \longrightarrow A b$, which maps an extension (1) onto the abelian group

$$
\frac{\mathfrak{h}_{\mathbb{Z}}}{\mathfrak{h}_{\mathbb{Z}}^{2}+(\mathbb{Z} H \cap[\mathbb{Z} G, \mathbb{Z} G])},
$$

where $\mathfrak{h}_{\mathbb{Z}}$ is the augmentation ideal of $\mathbb{Z} H$, and a morphism (2) onto the induced additive map

$$
\varphi_{*}: \frac{\mathfrak{h}_{\mathbb{Z}}}{\mathfrak{h}_{\mathbb{Z}}^{2}+(\mathbb{Z} H \cap[\mathbb{Z} G, \mathbb{Z} G])} \longrightarrow \frac{\mathfrak{h}_{\mathbb{Z}}^{\prime}}{\mathfrak{h}_{\mathbb{Z}}^{\prime 2}+\left(\mathbb{Z} H^{\prime} \cap\left[\mathbb{Z} G^{\prime}, \mathbb{Z} G^{\prime}\right]\right)} .
$$

In particular, there are isomorphisms $\lim _{\longleftarrow} \Lambda^{\prime} \simeq \lim \Lambda \simeq H_{2}(\Pi, \mathbb{Z})$.

(ii) If $k$ is a commutative ring, then we may define a functor $\Lambda_{k}^{\prime}$ in analogy with the functor $\Lambda^{\prime}$ defined in (i) above, by considering group rings with coefficients in $k$ instead of $\mathbb{Z}$. In the special case where $k$ is a field of characteristic 0 , we shall relate below the functor $\Lambda_{k}^{\prime}$ and certain higher order versions of it that are obtained by considering powers of the relevant augmentation ideals to the even homology groups of $\Pi$ with coefficients in $k$ (cf. Theorem 5.5 and Remark 5.6(ii)).

\section{Group homology as an inverse limit}

Let $\Pi$ be a group. Our goal in this section is to describe the homology groups of $\Pi$ with coefficients in a field $k$ of characteristic 0 as the inverse limit of suitable functors on the category of extensions $\mathfrak{E}_{g r p}(\Pi)$.

\subsection{Odd group homology}

We fix a non-negative integer $n$ and define two functors $Q_{2 n+1}$ and $P_{2 n+1}$ from $\mathfrak{E}_{g r p}(\Pi)$ to the category of $k$-vector spaces, as follows:

- The functor $Q_{2 n+1}$ maps a group extension (1) onto the vector space $I^{n+1} /\left[I, I^{n}\right]$, where $I=\mathfrak{h} \cdot k G$, while a morphism of extensions (2) is mapped onto the induced linear map $\varphi_{*}: I^{n+1} /\left[I, I^{n}\right] \longrightarrow I^{\prime n+1} /\left[I^{\prime}, I^{\prime n}\right]$, where $I^{\prime}=\mathfrak{h}^{\prime}$. $k G^{\prime}$.

- The functor $P_{2 n+1}$ maps a group extension (1) onto $\mathfrak{h}^{n+1} /\left(\mathfrak{h}^{n+1} \cap\left[I, I^{n}\right]\right)$, where $I=\mathfrak{h} \cdot k G$, while a morphism of extensions (2) is mapped onto the induced linear map $\varphi_{*}: \mathfrak{h}^{n+1} /\left(\mathfrak{h}^{n+1} \cap\left[I, I^{n}\right]\right) \longrightarrow \mathfrak{h}^{\prime n+1} /\left(\mathfrak{h}^{\prime n+1} \cap\left[I^{\prime}, I^{\prime n}\right]\right)$, where $I^{\prime}=\mathfrak{h}^{\prime} \cdot k G^{\prime}$.

We now define two natural transformations

$$
\eta_{2 n+1}: P_{2 n+1} \longrightarrow Q_{2 n+1} \text { and } \theta_{2 n+1}: Q_{2 n+1} \longrightarrow P_{2 n+1},
$$

by letting their components corresponding to the extension (1) be the linear maps ${ }^{1}$

$$
\eta_{2 n+1}: \mathfrak{h}^{n+1} /\left(\mathfrak{h}^{n+1} \cap\left[I, I^{n}\right]\right) \longrightarrow I^{n+1} /\left[I, I^{n}\right]
$$

\footnotetext{
${ }^{1}$ In order to simplify the notation, we use the same symbols for the components of the natural transformations $\eta_{2 n+1}$ and $\theta_{2 n+1}$.
} 
and

$$
\theta_{2 n+1}: I^{n+1} /\left[I, I^{n}\right] \longrightarrow \mathfrak{h}^{n+1} /\left(\mathfrak{h}^{n+1} \cap\left[I, I^{n}\right]\right),
$$

which are defined as follows: The map $\eta_{2 n+1}$ is induced by the inclusion $\mathfrak{h} \hookrightarrow \mathfrak{h}$. $k G=I$, while $\theta_{2 n+1}$ is induced by the map $\nu: k G \longrightarrow k H$, which maps any element $\sum_{g \in G} a_{g} g \in k G$ onto the element $\sum_{g \in H} a_{g} g \in k H$. As shown in [10, Thm. 2], these maps are well-defined and the composition $\theta_{2 n+1} \circ \eta_{2 n+1}$ is the identity on $\mathfrak{h}^{n+1} /\left(\mathfrak{h}^{n+1} \cap\left[I, I^{n}\right]\right)$. In order to verify the naturality of $\eta_{2 n+1}$ and $\theta_{2 n+1}$, we note that, given any morphism (2) of extensions, there are induced commutative diagrams

$$
\begin{aligned}
& \mathfrak{h} \hookrightarrow \mathfrak{h} \cdot k G \quad k G \stackrel{\nu}{\longrightarrow} k H \\
& \widetilde{\varphi} \downarrow \quad \downarrow \widetilde{\varphi} \quad \text { and } \quad \widetilde{\varphi} \downarrow \quad \downarrow \widetilde{\varphi} \\
& \mathfrak{h} \hookrightarrow \mathfrak{h}^{\prime} \cdot k G^{\prime} \quad k G^{\prime} \stackrel{\nu^{\prime}}{\longrightarrow} k H^{\prime}
\end{aligned}
$$

where the vertical arrows $\widetilde{\varphi}$ are induced by the group homomorphism $\varphi$ and $\nu^{\prime}$ is the linear map defined in analogy with $\nu$.

The arguments of $[\mathbf{1 0}, \S 3]$ show that the linear maps $\eta_{2 n+1}$ and $\theta_{2 n+1}$ are compatible with the Burghelea decomposition of the cyclic homology group $H C_{2 n+1}(k \Pi)$, in the sense that there is a commutative diagram

$$
\begin{array}{ccccc}
H C_{2 n+1}(k \Pi)_{[1]} & \longrightarrow & H C_{2 n+1}(k \Pi) & \longrightarrow & H C_{2 n+1}(k \Pi)_{[1]} \\
\gamma_{2 n+1,[1]} \downarrow & & \gamma_{2 n+1} \downarrow & & \gamma_{2 n+1,[1]} \downarrow \\
\mathfrak{h}^{n+1} /\left(\mathfrak{h}^{n+1} \cap\left[I, I^{n}\right]\right) & \stackrel{\eta_{2 n+1}}{\longrightarrow} & I^{n+1} /\left[I, I^{n}\right] & \stackrel{\theta_{2 n+1}}{\longrightarrow} & \mathfrak{h}^{n+1} /\left(\mathfrak{h}^{n+1} \cap\left[I, I^{n}\right]\right) .
\end{array}
$$

Here, $\gamma_{2 n+1}$ is the odd Connes homomorphism, $\gamma_{2 n+1,[1]}$ is the composition

$$
H C_{2 n+1}(k \Pi)_{[1]} \hookrightarrow H C_{2 n+1}(k \Pi) \stackrel{\gamma_{2 n+1}}{\longrightarrow} I^{n+1} /\left[I, I^{n}\right] \stackrel{\theta_{2 n+1}}{\longrightarrow} \mathfrak{h}^{n+1} /\left(\mathfrak{h}^{n+1} \cap\left[I, I^{n}\right]\right)
$$

and the unlabelled horizontal arrows are the canonical inclusion and projection respectively associated with the component $H C_{2 n+1}(k \Pi)_{[1]}$ in the Burghelea decomposition of the cyclic homology group $H C_{2 n+1}(k \Pi)$. Identifying the component $H C_{2 n+1}(k \Pi)_{[1]}$ with the vector space $H_{2 n+1}(\Pi, k) \oplus \cdots \oplus H_{3}(\Pi, k) \oplus H_{1}(\Pi, k)$, we obtain the linear map

$$
\gamma_{2 n+1,[1]}: H_{2 n+1}(\Pi, k) \oplus \cdots \oplus H_{3}(\Pi, k) \oplus H_{1}(\Pi, k) \longrightarrow \mathfrak{h}^{n+1} /\left(\mathfrak{h}^{n+1} \cap\left[I, I^{n}\right]\right) .
$$

This map is natural with respect to the extension (1) of the group $\Pi$ and hence there is an induced linear map

$$
\gamma_{2 n+1,[1]}: H_{2 n+1}(\Pi, k) \oplus \cdots \oplus H_{3}(\Pi, k) \oplus H_{1}(\Pi, k) \longrightarrow \lim _{\longleftarrow} P_{2 n+1} .
$$

Proposition 5.1. The linear map (10) defined above is surjective for all $n \geqslant 1$, while its kernel is identified with the group $H_{1}(\Pi, k)$ for all $n \geqslant 0$.

Proof. We fix a free presentation (8) of the group $\Pi$ and let $I=\mathfrak{n} \cdot k F$. Then, in view of Example 4.1 and Lemma 4.2, the inverse limit $\lim _{2} P_{2 n+1}$ is the subspace of $\mathfrak{n}^{n+1} /\left(\mathfrak{n}^{n+1} \cap\left[I, I^{n}\right]\right)$, consisting of those vectors that are equalized by the linear maps induced by all parallel pairs of morphisms from the free presentation (8); let $\xi \in \mathfrak{n}^{n+1} /\left(\mathfrak{n}^{n+1} \cap\left[I, I^{n}\right]\right)$ be such a vector. In view of the naturality of $\eta_{2 n+1}$, the vector $\eta_{2 n+1}(\xi) \in I^{n+1} /\left[I, I^{n}\right]$ is also equalized by the linear maps induced by all 
parallel pairs of morphisms from the free presentation (8). Then, Corollary 1.3 implies that $\eta_{2 n+1}(\xi)$ is equalized by the linear maps induced by the parallel pair of morphisms

$$
u, v: k F \longrightarrow k F \oplus \Omega_{k F}^{1},
$$

defined in Remark 2.3(i). Assuming that $n \geqslant 1$, it follows from loc. cit. that

$$
\eta_{2 n+1}(\xi) \in \operatorname{im}\left[H C_{2 n+1}(k \Pi) \stackrel{\gamma_{2 n+1}}{\longrightarrow} I^{n+1} /\left[I, I^{n}\right]\right] .
$$

Invoking the commutativity of the right-hand square in diagram (9), we conclude that the element $\xi=\theta_{2 n+1}\left(\eta_{2 n+1}(\xi)\right)$ is contained in the image of the map

$$
\gamma_{2 n+1,[1]}: H C_{2 n+1}(k \Pi)_{[1]} \longrightarrow \mathfrak{n}^{n+1} /\left(\mathfrak{n}^{n+1} \cap\left[I, I^{n}\right]\right) .
$$

Therefore, the linear map (10) is surjective for $n \geqslant 1$. Its kernel equals $H_{1}(\Pi, k)$ for all $n \geqslant 0$, in view of Remark 2.3(ii) and the commutativity of the left-hand square in diagram (9).

For any positive integer $n$ there is a natural transformation of functors

$$
\lambda_{2 n+1}: P_{2 n+1} \longrightarrow P_{2 n-1},
$$

whose component on the extension (1) of $\Pi$ is the linear map

$$
\lambda_{2 n+1}: \mathfrak{h}^{n+1} /\left(\mathfrak{h}^{n+1} \cap\left[I, I^{n}\right]\right) \longrightarrow \mathfrak{h}^{n} /\left(\mathfrak{h}^{n} \cap\left[I, I^{n-1}\right]\right),
$$

which is induced by the inclusion $\mathfrak{h}^{n+1} \hookrightarrow \mathfrak{h}^{n}$. The functor

$$
\Lambda_{2 n+1}=\operatorname{ker} \lambda_{2 n+1}
$$

associates with the extension (1) the vector space

$$
\left(\mathfrak{h}^{n+1} \cap\left[I, I^{n-1}\right]\right) /\left(\mathfrak{h}^{n+1} \cap\left[I, I^{n}\right]\right),
$$

whereas a morphism (2) is mapped onto the induced linear map

$$
\varphi_{*}:\left(\mathfrak{h}^{n+1} \cap\left[I, I^{n-1}\right]\right) /\left(\mathfrak{h}^{n+1} \cap\left[I, I^{n}\right]\right) \longrightarrow\left(\mathfrak{h}^{\prime n+1} \cap\left[I^{\prime}, I^{\prime n-1}\right]\right) /\left(\mathfrak{h}^{\prime n+1} \cap\left[I^{\prime}, I^{\prime n}\right]\right) .
$$

We note that for all $n \geqslant 1$ there is a commutative diagram

$$
\begin{array}{ccccc}
H C_{2 n+1}(k \Pi)_{[1]} & \hookrightarrow H C_{2 n+1}(k \Pi) & \stackrel{\gamma_{2 n+1}}{\longrightarrow} & I^{n+1} /\left[I, I^{n}\right] \stackrel{\theta_{2 n+1}}{\longrightarrow} \mathfrak{h}^{n+1} /\left(\mathfrak{h}^{n+1} \cap\left[I, I^{n}\right]\right) \\
\quad(n+1) S \downarrow & (n+1) S \downarrow & & \downarrow & \\
H C_{2 n-1}(k \Pi)_{[1]} & \hookrightarrow H C_{2 n-1}(k \Pi) & \stackrel{\gamma_{2 n-1}}{\longrightarrow} & I^{n} /\left[I, I^{n-1}\right] \stackrel{\theta_{2 n+1}}{\longrightarrow} & \mathfrak{h}^{n} /\left(\mathfrak{h}^{n} \cap\left[I, I^{n-1}\right]\right)
\end{array}
$$

where $S$ is the Connes periodicity operator and the unlabelled vertical arrow is induced by the inclusion $I^{n+1} \hookrightarrow I^{n}$. The commutativity of the square in the middle follows from Lemma 3.1. Taking into account the behavior of $S$ on the component $H C_{2 n+1}(k \Pi)_{[1]}$ of $H C_{2 n+1}(k \Pi)$, we conclude that the image of the subspace $H_{2 n+1}(\Pi, k) \subseteq H C_{2 n+1}(k \Pi)_{[1]}$ under the map

$$
\gamma_{2 n+1,[1]}: H C_{2 n+1}(k \Pi)_{[1]} \longrightarrow \mathfrak{h}^{n+1} /\left(\mathfrak{h}^{n+1} \cap\left[I, I^{n}\right]\right)
$$

is contained in the kernel of the map (11). We may therefore define the linear map

$$
\beta_{2 n+1}: H_{2 n+1}(\Pi, k) \longrightarrow\left(\mathfrak{h}^{n+1} \cap\left[I, I^{n-1}\right]\right) /\left(\mathfrak{h}^{n+1} \cap\left[I, I^{n}\right]\right)
$$


by restricting $\gamma_{2 n+1,[1]}$. This map depends naturally upon the extension (1) of $\Pi$; hence, there is an induced linear map

$$
\beta_{2 n+1}: H_{2 n+1}(\Pi, k) \longrightarrow \lim _{\longleftarrow} \Lambda_{2 n+1} .
$$

Theorem 5.2. The linear map (13) defined above is an isomorphism for all $n \geqslant 1$.

Proof. We consider the diagram

$$
\begin{aligned}
& 0 \longrightarrow H_{2 n+1}(\Pi, k) \longrightarrow H C_{2 n+1}(k \Pi)_{[1]} \stackrel{(n+1) S}{\longrightarrow} H C_{2 n-1}(k \Pi)_{[1]} \longrightarrow 0 \\
& 0 \quad \longrightarrow \quad \underline{\lim } \Lambda_{2 n+1} \quad \longrightarrow \quad \underline{\lim } P_{2 n+1} \quad \stackrel{\lambda_{2 n+1}}{\longrightarrow} \quad \underline{\lim } P_{2 n-1} .
\end{aligned}
$$

The left-hand square is commutative in view of the definition of $\beta_{2 n+1}$ and the commutativity of the right-hand square follows from the commutativity of diagram (12). The top row of the diagram is obviously exact, and the exactness of the bottom row follows from the left exactness of the inverse limit functor, in view of the exact sequence of functors

$$
0 \longrightarrow \Lambda_{2 n+1} \longrightarrow P_{2 n+1} \stackrel{\lambda_{2 n+1}}{\longrightarrow} P_{2 n-1}
$$

(cf. Lemma 4.3). Since the maps $\gamma_{2 n+1,[1]}$ and $\gamma_{2 n-1,[1]}$ have kernel $H_{1}(\Pi, k)$, and $\gamma_{2 n+1,[1]}$ is surjective (cf. Proposition 5.1), the result follows by applying the snake lemma.

In the special case where $n=1$, the following result was noted in $[\mathbf{1 0}$, Thm. 4].

Corollary 5.3. Consider a free presentation (8) of the group $\Pi$ and the ideal $I=$ $\mathfrak{n} \cdot k F \subseteq k F$. Then the linear map

$$
\beta_{2 n+1}: H_{2 n+1}(\Pi, k) \longrightarrow\left(\mathfrak{n}^{n+1} \cap\left[I, I^{n-1}\right]\right) /\left(\mathfrak{n}^{n+1} \cap\left[I, I^{n}\right]\right)
$$

is injective for all $n \geqslant 1$.

Proof. This follows from Theorem 5.2, in view of Example 4.1 and Lemma 4.2.

\subsection{Even group homology}

The approach is completely analogous to the odd case. We fix a non-negative integer $n$ and define two functors $Q_{2 n}$ and $P_{2 n}$ from $\mathfrak{E}_{g r p}(\Pi)$ to the category of $k$-vector spaces, as follows:

- The functor $Q_{2 n}$ maps a group extension (1) onto $k G /\left([k G, k G]+I^{n+1}\right)$, where $I=\mathfrak{h} \cdot k G$, while a morphism of extensions (2) is mapped onto the induced linear map $\varphi_{*}: k G /\left([k G, k G]+I^{n+1}\right) \longrightarrow k G^{\prime} /\left(\left[k G^{\prime}, k G^{\prime}\right]+I^{\prime n+1}\right)$, where $I^{\prime}=$ $\mathfrak{h}^{\prime} \cdot k G^{\prime}$.

- The functor $P_{2 n}$ maps a group extension (1) onto the vector space $k H /\left(\mathfrak{h}^{n+1}+\right.$ $B$ ), where $B=k H \cap[k G, k G]$, while a morphism of extensions (2) is mapped onto the induced linear map $\varphi_{*}: k H /\left(\mathfrak{h}^{n+1}+B\right) \longrightarrow k H^{\prime} /\left(\mathfrak{h}^{\prime n+1}+B^{\prime}\right)$, where $B^{\prime}=k H^{\prime} \cap\left[k G^{\prime}, k G^{\prime}\right]$. 
We now define two natural transformations

$$
\eta_{2 n}: P_{2 n} \longrightarrow Q_{2 n} \text { and } \theta_{2 n}: Q_{2 n} \longrightarrow P_{2 n}
$$

by letting their components corresponding to the extension (1) be the linear maps

$$
\eta_{2 n}: k H /\left(\mathfrak{h}^{n+1}+B\right) \longrightarrow k G /\left([k G, k G]+I^{n+1}\right)
$$

and

$$
\theta_{2 n}: k G /\left([k G, k G]+I^{n+1}\right) \longrightarrow k H /\left(\mathfrak{h}^{n+1}+B\right),
$$

which are defined as follows: The map $\eta_{2 n}$ is induced by the inclusion $k H \hookrightarrow k G$, while $\theta_{2 n}$ is induced by the map $\nu: k G \longrightarrow k H$, which maps any element $\sum_{g \in G} a_{g} g \in k G$ onto the element $\sum_{g \in H} a_{g} g \in k H$. As shown in [10, Thm. 1], these maps are well-defined and the composition $\theta_{2 n} \circ \eta_{2 n}$ is the identity on $k H /\left(\mathfrak{h}^{n+1}+B\right)$.

\begin{tabular}{|c|c|c|c|c|}
\hline$\hookrightarrow$ & $k G$ & & $k G$ & $\stackrel{\nu}{\longrightarrow}$ \\
\hline 1 & $\downarrow \widetilde{\varphi}$ & and & $\widetilde{\varphi} \downarrow$ & \\
\hline$H^{\prime} \hookrightarrow$ & $k G^{\prime}$ & & $k G^{\prime}$ & $\stackrel{\nu^{\prime}}{\longrightarrow}$ \\
\hline
\end{tabular}
In order to verify the naturality of $\eta_{2 n}$ and $\theta_{2 n}$, we note that given a morphism (2) of extensions, there are induced commutative diagrams

where the vertical arrows $\widetilde{\varphi}$ are induced by the group homomorphism $\varphi$ and $\nu^{\prime}$ is the linear map defined in analogy with $\nu$.

The arguments of $[\mathbf{1 0}, \S 3]$ show that the linear maps $\eta_{2 n}$ and $\theta_{2 n}$ are compatible with the Burghelea decomposition of the cyclic homology group $H C_{2 n}(k \Pi)$, in the sense that there is a commutative diagram

$$
\begin{array}{ccccc}
H C_{2 n}(k \Pi)_{[1]} & \longrightarrow & H C_{2 n}(k \Pi) & \longrightarrow & H C_{2 n}(k \Pi)_{[1]} \\
\gamma_{2 n,[1]} \downarrow & & \gamma_{2 n} \downarrow & & \gamma_{2 n,[1]} \downarrow \\
k H /\left(\mathfrak{h}^{n+1}+B\right) & \stackrel{\eta_{2 n}}{\longrightarrow} & k G /\left([k G, k G]+I^{n+1}\right) & \stackrel{\theta_{2 n}}{\longrightarrow} & k H /\left(\mathfrak{h}^{n+1}+B\right) .
\end{array}
$$

Here, $\gamma_{2 n}$ is the even Connes homomorphism, $\gamma_{2 n,[1]}$ is the composition

$$
H C_{2 n}(k \Pi)_{[1]} \hookrightarrow H C_{2 n}(k \Pi) \stackrel{\gamma_{2 n}}{\longrightarrow} k G /\left([k G, k G]+I^{n+1}\right) \stackrel{\theta_{2 n}}{\longrightarrow} k H /\left(\mathfrak{h}^{n+1}+B\right)
$$

and the unlabelled horizontal arrows are the canonical inclusion and projection respectively associated with the component $H C_{2 n}(k \Pi)_{[1]}$ in the Burghelea decomposition of the cyclic homology group $H C_{2 n}(k \Pi)$. Identifying the component $H C_{2 n}(k \Pi)_{[1]}$ with the vector space $H_{2 n}(\Pi, k) \oplus \cdots \oplus H_{2}(\Pi, k) \oplus H_{0}(\Pi, k)$, we obtain the linear map

$$
\gamma_{2 n,[1]}: H_{2 n}(\Pi, k) \oplus \cdots \oplus H_{2}(\Pi, k) \oplus H_{0}(\Pi, k) \longrightarrow k H /\left(\mathfrak{h}^{n+1}+B\right) .
$$

This map is natural with respect to the extension (1) of the group $\Pi$ and hence there is an induced linear map

$$
\gamma_{2 n,[1]}: H_{2 n}(\Pi, k) \oplus \cdots \oplus H_{2}(\Pi, k) \oplus H_{0}(\Pi, k) \longrightarrow \underset{\longleftarrow}{\lim } P_{2 n} .
$$

Proposition 5.4. The linear map (15) defined above is an isomorphism for all $n \geqslant 0$.

Proof. We fix a free presentation (8) of the group $\Pi$ and let $I=\mathfrak{n} \cdot k F$. Then, in view of Example 4.1 and Lemma 4.2, the inverse $\operatorname{limit} \lim P_{2 n}$ is the subspace of 
$k N /\left(\mathfrak{n}^{n+1}+B\right)$, consisting of those vectors that are equalized by the linear maps induced by all parallel pairs of morphisms from the free presentation (8); let us consider such a vector $\xi \in k N /\left(\mathfrak{n}^{n+1}+B\right)$. In view of the naturality of $\eta_{2 n}$, the vector $\eta_{2 n}(\xi) \in$ $k F /\left([k F, k F]+I^{n+1}\right)$ is also equalized by the linear maps induced by all parallel pairs of morphisms from the free presentation (8). Then, Corollary 1.3 implies that $\eta_{2 n}(\xi)$ is equalized by the linear maps induced by the parallel pair of morphisms

$$
u, v: k F \longrightarrow k F \oplus \Omega_{k F}^{1}
$$

of Remark 2.5. It follows from loc. cit. that

$$
\eta_{2 n}(\xi) \in \operatorname{im}\left[H C_{2 n}(k \Pi) \stackrel{\gamma_{2 n}}{\longrightarrow} k F /\left([k F, k F]+I^{n+1}\right)\right] .
$$

Invoking the commutativity of the right-hand square in diagram (14), we conclude that the element $\xi=\theta_{2 n}\left(\eta_{2 n}(\xi)\right)$ is contained in the image of the map

$$
\gamma_{2 n,[1]}: H C_{2 n}(k \Pi)_{[1]} \longrightarrow k N /\left(\mathfrak{n}^{n+1}+B\right) .
$$

Therefore, the linear map (15) is surjective. Its injectivity follows from the exact sequence of Theorem 2.4, in view of the commutativity of the left-hand square in diagram (14).

For any positive integer $n$ there is a natural transformation of functors

$$
\lambda_{2 n}: P_{2 n} \longrightarrow P_{2 n-2},
$$

whose component on the extension (1) of $\Pi$ is the linear map

$$
\lambda_{2 n}: k H /\left(\mathfrak{h}^{n+1}+B\right) \longrightarrow k H /\left(\mathfrak{h}^{n}+B\right),
$$

which is induced by the identity map of $k H$. The functor

$$
\Lambda_{2 n}=\operatorname{ker} \lambda_{2 n}
$$

associates with the extension (1) of $\Pi$ the vector space

$$
\left(\mathfrak{h}^{n}+B\right) /\left(\mathfrak{h}^{n+1}+B\right),
$$

while a morphism (2) is mapped onto the induced linear map

$$
\varphi_{*}:\left(\mathfrak{h}^{n}+B\right) /\left(\mathfrak{h}^{n+1}+B\right) \longrightarrow\left(\mathfrak{h}^{\prime n}+B^{\prime}\right) /\left(\mathfrak{h}^{\prime n+1}+B^{\prime}\right) .
$$

We note that for all $n \geqslant 1$ there is a commutative diagram

$$
\begin{aligned}
& H C_{2 n}(k \Pi)_{[1]} \hookrightarrow H C_{2 n}(k \Pi) \stackrel{\gamma_{2 n}}{\longrightarrow} k G /\left([k G, k G]+I^{n+1}\right) \stackrel{\theta_{2 n}}{\longrightarrow} k H /\left(\mathfrak{h}^{n+1}+B\right) \\
& (n+1) S \downarrow \quad(n+1) S \downarrow \quad \downarrow \quad \downarrow \lambda_{2 n} \\
& H C_{2 n-2}(k \Pi)_{[1]} \hookrightarrow H C_{2 n-2}(k \Pi) \stackrel{\gamma_{2 n-2}}{\longrightarrow} k G /\left([k G, k G]+I^{n}\right) \stackrel{\theta_{2 n-2}}{\longrightarrow} k H /\left(\mathfrak{h}^{n}+B\right)
\end{aligned}
$$

where $S$ is the Connes periodicity operator and the unlabelled vertical arrow is induced by the identity of $k G$. The commutativity of the square in the middle follows from Lemma 3.2. Taking into account the behavior of $S$ on the component $H C_{2 n}(k \Pi)_{[1]}$ of $H C_{2 n}(k \Pi)$, we conclude that the image of the subspace $H_{2 n}(\Pi, k) \subseteq H C_{2 n}(k \Pi)_{[1]}$ 
under the map

$$
\gamma_{2 n,[1]}: H C_{2 n}(k \Pi)_{[1]} \longrightarrow k H /\left(\mathfrak{h}^{n+1}+B\right)
$$

is contained in the kernel of the map (16). We may therefore define the linear map

$$
\beta_{2 n}: H_{2 n}(\Pi, k) \longrightarrow\left(\mathfrak{h}^{n}+B\right) /\left(\mathfrak{h}^{n+1}+B\right)
$$

by restricting $\gamma_{2 n,[1]}$. This map depends naturally upon the extension (1) of $\Pi$; hence there is an induced linear map

$$
\beta_{2 n}: H_{2 n}(\Pi, k) \longrightarrow \lim _{\longleftarrow} \Lambda_{2 n} .
$$

Theorem 5.5. The linear map (18) defined above is an isomorphism for all $n \geqslant 1$.

Proof. We consider the diagram

$$
\begin{aligned}
& 0 \longrightarrow H_{2 n}(\Pi, k) \longrightarrow H C_{2 n}(k \Pi)_{[1]} \stackrel{(n+1) S}{\longrightarrow} H C_{2 n-2}(k \Pi)_{[1]} \longrightarrow 0 \\
& \beta_{2 n} \downarrow \quad \gamma_{2 n,[1]} \downarrow \quad \gamma_{2 n-2,[1]} \downarrow \\
& 0 \quad \longrightarrow \quad \lim _{2 n} \Lambda_{2 n} \quad \longrightarrow \quad \lim _{\longleftarrow} P_{2 n} \quad \stackrel{\lambda_{2 n}}{\longrightarrow} \quad \lim _{\longleftarrow} P_{2 n-2} .
\end{aligned}
$$

The left-hand square is commutative, in view of the definition of $\beta_{2 n}$, while the commutativity of the right-hand square follows from the commutativity of diagram (17). The top row of the diagram is obviously exact, while the exactness of the bottom row follows from the left exactness of the inverse limit functor, in view of the exact sequence of functors

$$
0 \longrightarrow \Lambda_{2 n} \longrightarrow P_{2 n} \stackrel{\lambda_{2 n}}{\longrightarrow} P_{2 n-2}
$$

(cf. Lemma 4.3). Since the maps $\gamma_{2 n,[1]}$ and $\gamma_{2 n-2,[1]}$ are both bijective (cf. Proposition 5.4), the result follows by applying the snake lemma.

Remark 5.6. Let $\Pi$ be a group.

(i) We consider a free presentation (8) of $\Pi$ and the subspace $B=k N \cap[k F, k F]$ $\subseteq k N$. Then, in view of Theorem 5.5, Example 4.1 and Lemma 4.2, the linear map

$$
\beta_{2 n}: H_{2 n}(\Pi, k) \longrightarrow\left(\mathfrak{n}^{n}+B\right) /\left(\mathfrak{n}^{n+1}+B\right)
$$

is injective for all $n \geqslant 1$. This assertion was noted in [10, Thm. 3].

(ii) Since $k H \cap[k G, k G] \subseteq \mathfrak{h}$ for any extension (1) of $\Pi$, the functor $\Lambda_{2}$ coincides with the functor $\Lambda_{k}^{\prime}$ defined in Remark 4.5(ii). In this way, Proposition 4.4 indicates that some of the results obtained in this section may be valid over more general coefficient rings as well.

\section{References}

[1] Brown, K.S., Cohomology of groups, Graduate Texts in Mathematics, vol. 87, Springer-Verlag, New York-Berlin, 1982.

[2] Burghelea, D., The cyclic homology of group rings, Comment. Math. Helv. 60 (1985), 354-365. 
[3] Connes, A., Non-commutative differential geometry, Publ. Math. Inst. Hautes Études Sci. 62 (1985), 41-144.

[4] Cuntz, J. and Quillen, D., Algebra extensions and non-singularity, J. Amer. Math. Soc. 8 (1995), 251-289.

[5] Emmanouil, I. and Mikhailov, R., A limit approach to group homology, J. Algebra 319 (2008), 1450-1461.

[6] Feigin, B.L. and Tsygan, B.L., Additive $K$-theory, $K$-theory, arithmetic and geometry (Moscow, 1984-1986), Lecture Notes in Mathematics, vol. 1289, Springer-Verlag, Berlin, 1987, 67-209.

[7] Gruenberg, K.W., Cohomological Topics in Group Theory, Lecture Notes in Mathematics, vol. 143, Springer-Verlag, Berlin-New York, 1970.

[8] Loday, J.-L., Cyclic Homology, Grundlehren der Mathematischen Wissenschaften [Fundamental Principles of Mathematical Sciences], SpringerVerlag, Berlin, 1992.

[9] MacLane, S., Categories for the Working Mathematician, Graduate Texts in Mathematics, vol. 5, Springer-Verlag, New York-Berlin, 1971.

[10] Mikhailov, R. and Passi, I.B.S., Higher traces on group rings. Comm. Algebra 33 (2005), 987-997.

[11] Quillen, D., Cyclic cohomology and algebra extensions. K-Theory 3 (1989), 205-246.

Ioannis Emmanouil emmanoui@math.uoa.gr

Department of Mathematics, University of Athens, Athens 15784, Greece

Inder Bir S. Passi ibspassi@yahoo.co.in

Senior Scientist, INSA, Centre for Advanced Study in Mathematics, Panjab University, Chandigarh 160014, India 